\title{
Higher reasoning with level-2 fuzzy regions
}

\author{
Jörg Verstraete ${ }^{1,2}$ \\ 1 Systems Research Institute - Polish Academy of Sciences \\ Ul. Newelska 6, 01-447 Warszawa, Poland \\ WWW home page: http://www.ibspan.waw.pl \\ jorg.verstraete@ibspan.waw.pl \\ 2 Database, Document and Content Management - Department of \\ Telecommunications and Information Processing \\ Sint Pietersnieuwstraat 41, 9000 Gent, Belgium \\ WWW home page: http://telin.ugent.be/ddcm \\ jorg.verstraete@telin.ugent.be
}

\begin{abstract}
Spatial data is quite often is prone to uncertainty and imprecision. For this purpose, fuzzy regions have been developed: they basically consist of a fuzzy set over a two dimensional domain, allowing for both fuzzy regions and fuzzy points to be modelled. The model is extended to a level-2 fuzzy region to overcome some limitations, but this has an impact on operations. In this contribution, we will look into the construction of and combination of existing data to yield level-2 fuzzy regions.
\end{abstract}

Keywords: level-2 fuzzy set, level-2 fuzzy region, fuzzy spatial

\section{Introduction}

A lot of data that is currently used involves some spatial content, and unfortunately this data often is prone to uncertainty and or imprecision: the origin for this imperfection can be the data itself (the real life data is inherently imperfect), limitations in measurements (the real life data is perfect, but it is impossible or too expensive to measure it accurately) or the combination of data (data from different sources can contradict or be incompatible). Applying flexible querying on crisp spatial data can also result in uncertain or imprecise results: a user can query for locations at walking distance, locations that involve steep inclines or locations where it is likely to see a specific animal or plant. Especially in the case where the data in the database is uncertain or imprecise, but even in the case where the data is crisp and flexible querying is permitted on spatial data, it is necessary to consider data structures capable of handling uncertain and imprecise spatial data. This contribution concerns the further development of the mathematical foundation of a spatial data structure capable of handling uncertainty and imprecision in spatial data. The structure is based on a simpler model from which implementable models have been derived.

Traditionally, there are two big types of models used for spatial data: entity based and field based. In an entity based approach, basic geometrical shapes are 
used to represent features: lines represent roads (or sides of the road); polygons can represent buildings or pieces of land. The field based approach allows numerical data to be represented over a region over interest, and is commonly used for e.g. pollution data: a grid is overlayed with the map, and with each cell of the grid a value is associated; this value is deemed representative for the area covered by the cell.

When representing fuzzy spatial data in a database, it is not only necessary to have the adequate operators to combine different data as dictated by the theory, but it is also necessary to provide operators to construct data from components. These operations can be necessary when generating the data to be stored in the database, but also when answering queries to generate the results. In this contribution an entity based approach, where features are subject to imperfection: their outline or location (or both) can be uncertain or imprecise. For the representation of uncertain or imprecise spatial features, fuzzy regions have been developed; these fuzzy regions are basically a fuzzy set over a two dimensional domain; a veristic interpretation allows us to represent regions with an imperfect outline, a possibilistic interpretation will allow the representation of an uncertain point. This model has been extended to level-2 fuzzy regions to allow for the modelling of uncertainty and imprecision in a single unified model and to allow both interpretations at the same time. For this level-2 representation, a number of operators have been developed; in this contribution we will consider higher level operations necessary to construct and reason with these models. Section 2 explains the fuzzy regions; in section 3 the extension to level-2 fuzzy regions is explained. Section 4 concerns the main topic if this paper and explains which operations can be used to construct and reason with level-2 fuzzy regions. The conclusion summarizes the findings.

\section{Fuzzy regions}

When modelling real world entities, it is frequent for the real world entity not to have an accurate or certain definition. Examples of this can be the spread of a pollutant, with varying degrees of concentration or just simply the edge of a lake. When representing this as a region, there must be some means to indicate that some points belong to a lesser extent to the region than other points. Some authors have used additional boundaries to indicate the points that fully belong and the points that do not belong to the region (broad boundary model [1],eggyolk model [2]), but such models don't allow for further specification of points in between both boundaries. The models have only been used for topological purposes as well. Other models have been developed, to model these points more accurately (e.g. [3] [4]).

The fuzzy region model requires a different view on regions: rather than defining a region by means of a boundary, the region is considered as a set of point. This set can the be augmented to a fuzzy set, thus allowing each point to have a membership grade. An overview of this model was presented in [5]. 


\subsection{Simple fuzzy regions}

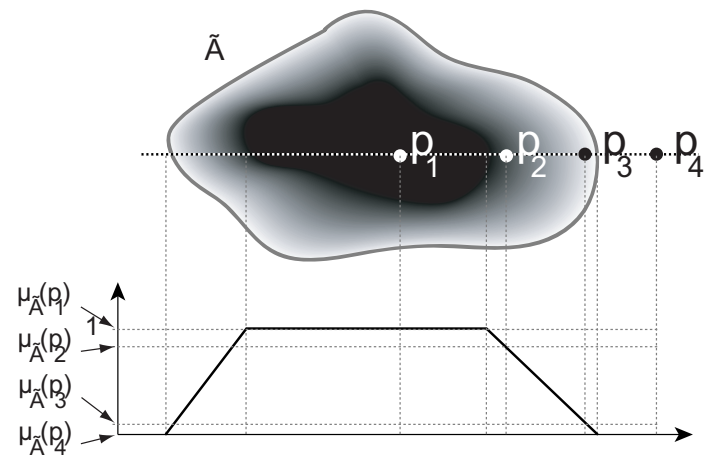

Fig. 1. The concept of a fuzzy region $\tilde{A}$; a fuzzy set over a two dimensional domain. All points belong to some extent to the region; indicated by means of the membership grade. The lower half of the figure shows a cross section. The shades of grey relate to the membership grades: darker shades match higher membership grades (the region has a dark outline to indicate its maximal outline).

\section{Definition 1 (Fuzzy region).}

$$
\tilde{R}=\left\{\left(p, \mu_{\tilde{R}}(p)\right) \mid p \in \mathbb{R}^{2}\right\}
$$

A fuzzy region essentially is a fuzzy set defined over a two dimensional domain; the concept is illustrated on figure 1. Consequently, the traditional fuzzy operations for intersection and union (t-norms and t-conorms) are immediately applicable.

\subsection{Fuzzy regions using powerset}

A first criticism to the fuzzy regions is that it is impossible to group point together. In some situations (e.g. the aforementioned example of the lake), it is possible that a user has additional knowledge that some points belong together and should form one basic element of the region. The fuzzy region model was therefore extended, by allowing the basic elements of the fuzzy region to be sets of points. This is illustrated on figure 2: The definition makes use of the powerset $^{3}$.

Definition 2 (Fuzzy region with powerset extension).

$$
\tilde{R}=\left\{\left(P, \mu_{\tilde{R}}(P)\right) \mid P \in \wp\left(\mathbb{R}^{2}\right) \wedge \forall P_{1}, P_{2} \in \tilde{R}: P_{1} \cap P_{2}=\emptyset\right\}
$$

\footnotetext{
${ }^{3}$ The powerset of a set is the set of all subsets contained in that one set, including the empty set and the set itself.
} 


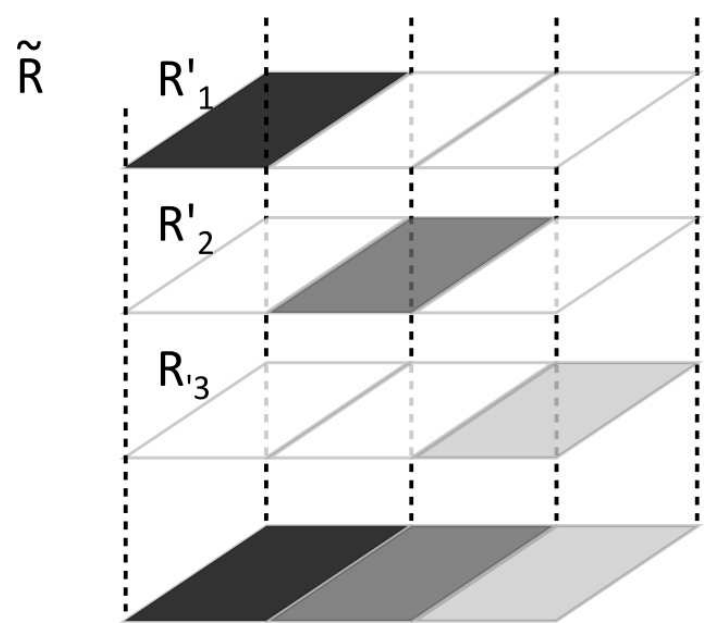

Fig. 2. A fuzzy region defined over the powerset of the two dimensional domain. The region $\tilde{R}$ is comprised of three elements, the regions $R_{1}^{\prime}, R_{2}^{\prime}$ and $R_{3}^{\prime}$ ). These are crisp regions that are given a membership grade with a veristic interpretation; they are elements or subregions.

This extension allows for individual points to be modelled. The fuzzy region allows for the representation of regions, when the fuzzy set is given a versitic interpretation: all points belong to some extent to the region. Not that the intersection of two basic elements is required to be empty; this limitation was made to facilitate the operations and has no real impact on the usefulness: the model was mainly considered as a stepping stone towards the level-2 fuzzy regions.

\section{Level-2 fuzzy regions}

Consider the example of the pollutant or the lake from section 2. Both of them could be represented using a fuzzy region (with a veristic interpretation), but interpretation may be slightly off. Do we consider a point that is definitely flooded (in the case of the lake) for certain water levels to partly belong? To further illustrate: what if we want to make statements? The water level of the lake is known to vary, can we represent the lake still as a fuzzy region? If we don't know the water level at some point in time, it would require a possibilistic interpretation to describe its boundary. Similarly, if we want to predict where the pollutant could be in the next few days, there may be different possibilities on how it spreads (depending on weather conditions for instance). So this basically yields a number of possible outlines and again requires a possibilistic interpretation. Furthermore, the current model is dependent on the metadata, stating if the fuzzy region has a possibilistic or a veristic interpretation. This knowledge is 
important in order to apply the correct operators, and it may be confusing and difficult to define operators between fuzzy regions with different interpretations.

These arguments led to the development of the level- 2 fuzzy regions. The level-2 fuzzy region uses a fuzzy region (as in definition 1 or 2 with a veristic interpretation as basic element. Several such fuzzy regions are combined in a new fuzzy set, in which they are given possibility degrees (this set thus has a possibilistic interpretation). Each fuzzy region represents a possibility for the (fuzzy) feature to be modelled; this is illustrated on figure 3. The different fuzzy

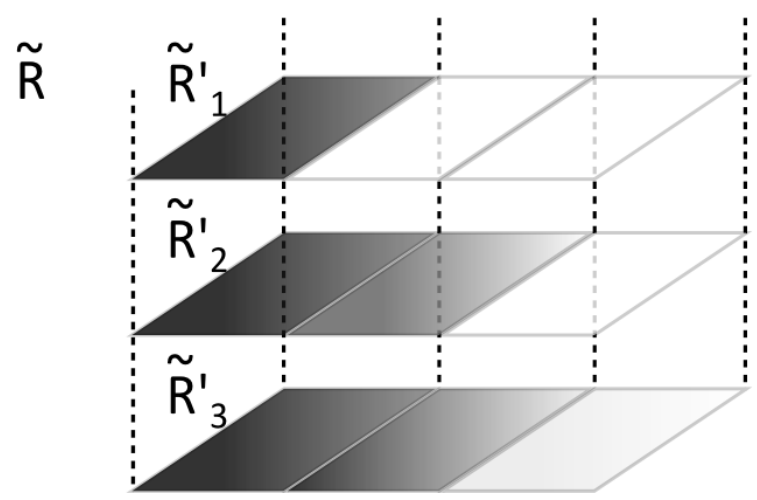

Fig. 3. The concept of a fuzzy region defined over the fuzzy powerset of the two dimensional domain. The region $\tilde{R}$ represents three possible candidates: the regions $\tilde{R}_{1}^{\prime}, \tilde{R}_{2}^{\prime}$ and $\left.\tilde{R}_{3}^{\prime}\right)$. These are fuzzy regions that are given a membership grade with a possibilistic interpretation; they are candidates or possibilities.

regions $\tilde{R}_{1}^{\prime}, \tilde{R}_{2}^{\prime}$ and $\tilde{R}_{3}^{\prime}$ could be representations for the lake with different water levels; or they could be predictions for the spread of the pollutant in different circumstances (e.g. different surface winds).

\subsection{Definition}

To achieve the concept formally, we will make use of the fuzzy powerset. The fuzzy powerset of a set $A$, denoted $\tilde{\wp}(A)$, is the set of all fuzzy subsets that belong to $A$. Using this concept, the level-2 fuzzy region can be defined over the $\tilde{\wp}\left(\mathbb{R}^{2}\right)$, effectively making the level-2 fuzzy region a fuzzy set of fuzzy sets (regions). This concept is known in literature as a level-2 fuzzy set ([6]) and is not to be confused with a type-2 fuzzy set ([7]), where the membership degrees are fuzzy sets. Simply stated, whereas the level-2 fuzzy set is a fuzzy set over a fuzzy domain with crisp membership grades, the type-2 fuzzy set is a fuzzy set over a crisp domain with fuzzy sets as membership grades. For extending the fuzzy regions; the level-2 concept was chosen as it allows us to model the concept of candidate fuzzy regions and keep the existing operations at that lower level. 
Using a type-2 extension would make it difficult to maintain the spatial relation between points in a concept similar to the candidate regions.

The level-2 fuzzy region can then be defined as:

$$
\tilde{R}=\left\{\left(\tilde{R}^{\prime}, \mu_{\tilde{R}}\left(\tilde{R}^{\prime}\right)\right) \mid \tilde{R}^{\prime} \in \tilde{\wp}\left(\mathbb{R}^{2}\right)\right\}
$$

with the membership function is defined as:

$$
\begin{aligned}
\mu_{\tilde{R}}: \tilde{\wp}\left(\mathbb{R}^{2}\right) & \mapsto[0,1] \\
\tilde{R}^{\prime} & \rightarrow \mu_{\tilde{R}}\left(\tilde{R}^{\prime}\right)
\end{aligned}
$$

Note that unlike in definition 2, the intersection of the elements is not required to be empty. This is because it concerns quite a different interpretation: in definition 2 the elements were considered to be subregions, groups of points that belong together; in the above definition, the elements are candidate regions. It is very likely for them to overlap in some parts, especially if the outline is not certain, but some central region is.

Operations In [8], obtaining information regarding points that belong to a level-2 fuzzy region has been considered. It was shown that the membership of a single point in the level-2 fuzzy region can be expressed by means of a type- 2 fuzzy set: each candidate region boasts a membership grade for the point; but with each candidate region comes a possibility degree. The union and intersection of level-2 fuzzy regions ([9]) has been presented; whereas other operations (e.g. distance and surface area) are under development.

\section{Combination of level-2 fuzzy regions}

Due to the nature of level-2 fuzzy regions, it is necessary to consider possible means of constructing them. This is illustrated on figure 4. The first idea would be to simply use the set operations (union, intersection). However, while the union of two level-2 fuzzy regions yields a new level-2 fuzzy region, it does not really allow for an easy creation of a level-2 fuzzy set given a number of possible fuzzy regions. For this purpose, we need to introduce additional operations. Similarly, the intersection of two level-2 fuzzy regions yields a new level-2 fuzzy region, but it does not provide for an easy way of reducing the number of possibilities.

We will first list the set operations, show their shortcomings for constructing level-2 fuzzy regions and then introduce the additional operations.

\subsection{Set operations}

In [9], the traditional set operations applied on level-2 fuzzy regions were presented; they require a double application of Zadehs extension principle ([10]), and the definitions will be repeated below. 
Union The union of two level-2 fuzzy regions is defined by means of the extension principle.

Definition 3 (Union of level-2 fuzzy regions).

$$
\tilde{R}_{1} \cup \tilde{R}_{2}=\bigcup_{R_{1}^{\prime}: \mu_{\left(R_{1}\right)}\left(\tilde{R}_{1}^{\prime}\right)>0 \wedge R_{2}^{\prime}: \mu_{\left(R_{2}\right)}\left(\tilde{R}_{2}^{\prime}\right)>0}\left\{\left(\tilde{R}_{1}^{\prime} \cup \tilde{R}_{2}^{\prime}, \mu_{\tilde{R_{1}} \cup \tilde{R_{2}}}\left(\tilde{R}_{1}^{\prime} \cup \tilde{R}_{2}^{\prime}\right)\right)\right\}
$$

The membership function is defined as:

$$
\begin{aligned}
\mu_{\tilde{R}}: \wp\left(\mathbb{R}^{2}\right) & \mapsto[0,1] \\
\mu_{\tilde{R_{1}} \cup \tilde{R_{2}}}\left(\tilde{R_{1}^{\prime}} \cup \tilde{R_{2}^{\prime}}\right) & \rightarrow S\left(\mu_{\left(R_{1}\right)}\left(\tilde{R}_{1}^{\prime}\right), \mu_{\left(R_{2}\right)}\left(\tilde{R}_{2}^{\prime}\right)\right)
\end{aligned}
$$

Conceptually, we see that the union takes all possible combinations of the candidate regions.

Intersection The intersection of two level-2 fuzzy regions is defined by means of the extension principle.

Definition 4 (Intersection of level-2 fuzzy regions).

$$
\tilde{R}_{1} \cap \tilde{R}_{2}=\bigcup_{R_{1}^{\prime}: \mu_{\left(R_{1}\right)}\left(\tilde{R}_{1}^{\prime}\right)>0 \wedge R_{2}^{\prime}: \mu_{\left(R_{2}\right)}\left(\tilde{R}_{2}^{\prime}\right)>0}\left\{\left(\tilde{R}_{1}^{\prime} \cap \tilde{R}_{2}^{\prime}, \mu_{\tilde{R_{1}} \cap \tilde{R_{2}}}\left(\tilde{R_{1}^{\prime}} \cap \tilde{R}_{2}^{\prime}\right)\right)\right\}
$$

The membership function is defined as:

$$
\begin{aligned}
\mu_{\tilde{R}}: \wp\left(\mathbb{R}^{2}\right) & \mapsto[0,1] \\
\mu_{\tilde{R_{1}} \cap \tilde{R}_{2}}\left(\tilde{R}_{1}^{\prime} \cap \tilde{R}_{2}^{\prime}\right) & \rightarrow T\left(\mu_{\left(R_{1}\right)}\left(\tilde{R}_{1}^{\prime}\right), \mu_{\left(R_{2}\right)}\left(\tilde{R}_{2}^{\prime}\right)\right)
\end{aligned}
$$

Example As an example, we will consider two regions as shown on figure 4a and figure 4 b. Region $\tilde{A}$ is a level-2 fuzzy region with two fuzzy candidate regions; Region $\tilde{B}$ is a level-2 fuzzy region with a single candidate region. The result of the intersection operator of regions $\tilde{A}$ and $\tilde{B}$ is shown on figure 4 c, the union is illustrated on figure $4 \mathrm{~d}$. Note that $\tilde{R}_{4}^{\prime}=\tilde{R}_{1}^{\prime} \cap \tilde{R}_{3}^{\prime}$ and $\tilde{R}_{5}^{\prime}=\tilde{R}_{2}^{\prime} \cap \tilde{R}_{3}^{\prime}$; whereas $\tilde{R}_{6}^{\prime}=\tilde{R}_{1}^{\prime} \cup \tilde{R}_{3}^{\prime}$ and $\tilde{R}_{7}^{\prime}=\tilde{R}_{2}^{\prime} \cup \tilde{R}_{3}^{\prime}$. These are the results as obtained from the set-operation defined above.

To reason with level-2 fuzzy regions, it is also necessary to be able to add or remove possibilities to/from a level-2 fuzzy region. Adding the region $\tilde{B}$ to $\tilde{A}$ then yields the result shown on figure 4e. The example shows that - while union and intersection are meaningful operators to combine different data - there also is a need for additional operations to process the level-2 fuzzy regions.

\subsection{Construction of and reasoning with level-2 regions}

As was explained in the previous section, the union and intersection as presented in [9] are not really suited to add or remove candidate regions, nor to merely 


\section{$\tilde{\AA}$}

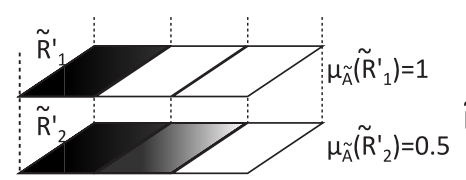

(a)

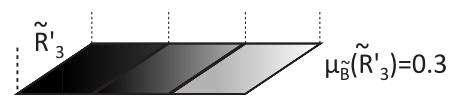

(b)

$\tilde{C}$

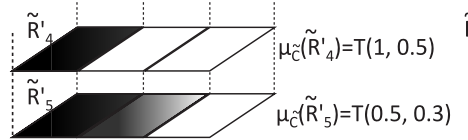

(c)

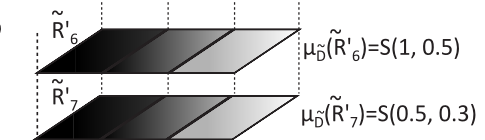

(d)

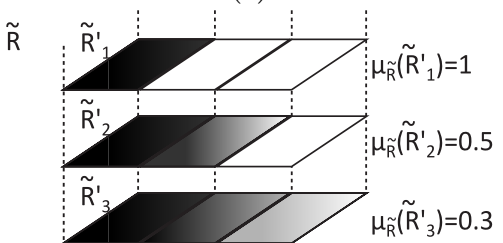

(e)

Fig. 4. An example showing the issues with union and intersection. Two 2 level-2 fuzzy regions are shown in (a) and (b), their intersection is shown in (c), their union in (d) and the combinations of both regions into a new level-2 fuzzy region in (d).

alter the possibilities of candidate regions. To increase or decrease the number of possibilities, it is not possible to use the standard union and intersection as defined above (and as suggested in [6]): the double application of the extension principle complicates things in this situation. For the purpose of easily modifying level-2 regions, additional operators will be introduced in this contribution. These operations are even considered by some as valid intersection and union operations, as the second application of the extension principle (as suggested in [6]) can yield counter-intuitive results.

Addition/Increase of possibilities To add candidate regions, we consider 2 level-2 fuzzy regions and merge all the candidate regions of both level-2 fuzzy regions. This means the union of only the top level needs to be considered. All possible candidate regions from both arguments are grouped in a single fuzzy set.

Definition 5 (Adding/increasing possibilities in level-2 fuzzy regions).

$$
\tilde{R}_{1} \oplus \tilde{R}_{2}=\bigcup_{R^{\prime}: \mu_{\tilde{R}_{1}}\left(\tilde{R}^{\prime}\right)>0 \vee \mu_{\tilde{R}_{2}}\left(\tilde{R}^{\prime}\right)>0}\left\{\left(\tilde{R}^{\prime}, \mu_{\tilde{R_{1}} \oplus \tilde{R_{2}}}\left(\tilde{R}^{\prime}\right)\right)\right\}
$$

The membership function is defined as:

$$
\begin{aligned}
\mu_{\tilde{R}}: \wp\left(\mathbb{R}^{2}\right) & \mapsto[0,1] \\
\mu_{\tilde{R}_{1} \oplus \tilde{R}_{2}}\left(\tilde{R}^{\prime}\right) & \rightarrow S\left(\mu_{\left(R_{1}\right)}\left(\tilde{R}^{\prime}\right), \mu_{\left(R_{2}\right)}\left(\tilde{R}^{\prime}\right)\right)
\end{aligned}
$$


If the region to be added occurs twice in the result, an appropriate T-conorm is used to determine the membership grade of the region - commonly, this will be the maximum. This allows for multiple information about different candidates for the same region to be combined. The result of this is illustrated on figure 4 .

Reduction of possibilities A similar approach can be applied to reduce the number of possibilities. Rather than consider all candidate regions that occur either fuzzy region, we only consider those that only belong to both candidate regions, thus limiting the number of possiblities and possibly decreasing the membership grades.

Definition 6 (Reduction of the possibilities in a level-2 fuzzy region).

$$
\tilde{R}_{1} \ominus \tilde{R_{2}}=\bigcup_{R^{\prime}: \mu_{\tilde{R_{1}}}\left(\tilde{R}^{\prime}\right)>0 \wedge R^{\prime}: \mu_{\tilde{R_{2}}}\left(\tilde{R}^{\prime}\right)>0}\left\{\left(\tilde{R}^{\prime}, \mu_{\tilde{R_{1}} \ominus \tilde{R_{2}}}\left(\tilde{R}^{\prime}\right)\right)\right\}
$$

The membership function is defined as:

$$
\begin{aligned}
\mu_{\tilde{R}}: \wp\left(\mathbb{R}^{2}\right) & \mapsto[0,1] \\
\mu_{\tilde{R_{1}} \ominus \tilde{R_{2}}}\left(\tilde{R}^{\prime}\right) & \rightarrow T\left(\mu_{\left(R_{1}\right)}\left(\tilde{R}^{\prime}\right), \mu_{\left(R_{2}\right)}\left(\tilde{R}^{\prime}\right)\right)
\end{aligned}
$$

If a candidate region $\tilde{R}^{\prime}$ exists in both level-2 fuzzy regions, a T-norm is used to determine its membership grade; this commonly will be the minimum. Note that to remove a candidate region, it should not belong to one of the arguments.

To remove a candidate region, given the candidate region to remove as an argument, one would need an operation such as the one defined below. Not only does it allow for the complete removal, but it also allows for decreasing the membership grades.

Definition 7 (Removal of possibilities from a level-2 fuzzy region).

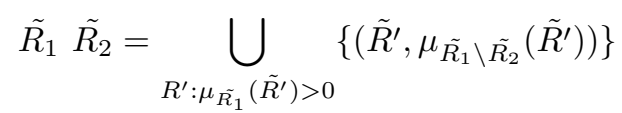

The membership function is defined as:

$$
\begin{aligned}
& \mu_{\tilde{R}}: \wp\left(\mathbb{R}^{2}\right) \mapsto[0,1] \\
& \mu_{\tilde{R}_{1} \tilde{R_{2}}}\left(\tilde{R}^{\prime}\right) \rightarrow \min \left(0, \mu_{\tilde{\left(R_{1}\right)}}\left(\tilde{R}^{\prime}\right)-\mu_{\left(R_{2}\right)}\left(\tilde{R}^{\prime}\right)\right)
\end{aligned}
$$

Complexity The model (and thus also the operations) at this point are purely theoretical to built the foundations for the representation models. To consider the complexity of the operators, first more practical models should be derived. This is currently in progress, and we are working on similar approaches as for the simple fuzzy regions: these were approximated using triangular networks or bitmaps $([5],[11],[12])$. The same representation can be used for the candidate fuzzy regions, in which case the complexity depends on both the representation methods uses and the number of candidate regions involved. As only a single application of the extension principle is performed, the operations will be faster than the set operations defined that apply the extension principle twice ([9]). 


\section{Application examples}

\subsection{Feature representation}

Consider a lake for which the water level can vary, based on the amount of rainfall of the last couple of weeks, melting snow at the end of winter, etc. Traditionally, a lake will be modelled by a single region; at best there can be flood regions associated with it. Using the level-2 fuzzy region model, it is possible to represent this lake as a single object in the database. The model can store as many different outlines for the lake as desired; these can be added to the model using the operator defined in Section 4.2. Any queries related to the lake will take into account all possible (represented) water levels in the results.

\subsection{Representation of query results}

As mentioned in the introduction, fuzzy queries on crisp data can yield fuzzy results. Querying a spatial database for locations that are close to an existing location is the simplest example. Similar results can be obtained for other queries. In an interface where the user indicates locations on a map, imprecision can be introduced to take into account the scale at which the user is working: if the user indicates a point on a map with a small scale, the point is likely to be less accurate than if a large scale would have been used.

\subsection{Usage in querying}

Suppose an area that matches specific criteria needs to be pinpointed: this can for instance be necessary to trace the last whereabouts of a missing person, or to determine the best place to build some facilities. Suppose some of the criteria are: close to a river, close to a highway, far from a forest, good connections with public transport, ... For every criteria, a level-2 fuzzy region can be constructed: close to a river will yield a number of possible locations; these can be combined with the results of the other queries using the intersection. Querying for a location that satisfies these criteria is then achieved by combining the level-2 fuzzy regions.

\section{Conclusion}

In this contribution, modification of level-2 fuzzy regions was considered. The level-2 fuzzy regions are a novel model to represent uncertainty and imprecision of spatial data represented by regions or points. The creation and modification of level-2 fuzzy regions is made more difficult by the fact that set operations mathematically need to be defined using a double application of the extension principle; this yields undesired results when intending to mereley add or remove possibilities. For this purpose, new operations have been introduced, which allow a more intuitive approach to add possibilities to or remove possibilities from a level-2 fuzzy set. 


\section{References}

1. Eliseo Clementini and Paolino Di Felice. An algebraic model for spatial objects with undetermined boundaries. GISDATA Specialist Meeting - revised version, 1994.

2. Anthony Cohn and Nicholas Mark Gotts. Spatial regions with undetermined boundaries. In Proceedings of the Second ACM Workshop on Advances in GIS, pages 52-59, 1994.

3. Markus Schneider. Geographic Objects with Indeterminate Boundaries, GISDATA Series, vol. 2, chapter Modelling Spatial Objects with Undetermined Boundaries Using the Realm/ROSE Approach, pages 141-152. Taylor \& Francis, 1996.

4. Virupaksha Kanjilal, Hechen Liu, and Markus Schneider. Plateau regions: An implementation concept for fuzzy regions in spatial databases and gis. In Eyke Hüllermeier, Rudolf Kruse, and Frank Hoffmann, editors, Computational Intelligence for Knowledge-Based Systems Design, 13th International Conference on Information Processing and Management of Uncertainty, IPMU 2010, Dortmund, Germany, June 28 - July 2, volume 6178 of Lecture Notes in Artificial Intelligence. Springer, 2010.

5. Jörg Verstraete, Guy De Tré, Rita De Caluwe, and Axel Hallez. Fuzzy modeling with Spatial Information for Geographic Problems; eds. Petry Fred, Robinson Vince, Cobb Maria, chapter Field based methods for the modelling of fuzzy spatial data., pages 41-69. Springer Verlag, 2005.

6. Siegried Gottwald. Set theory for fuzzy sets of higher level. Fuzzy sets and systems, 2(2):125-151, 1979.

7. Jerry M. Mendel. Uncertain rule-based fuzzy logic systems, Introduction and new directions. Prentice Hall, 2001.

8. Jörg Verstraete. Using level-2 fuzzy sets to combine uncertainty and imprecision in fuzzy regions. In E Mugellini, P.S. Szczepaniak, M. Chiara Pettenati, and M. Sokhn, editors, Advances in Intelligent Web Mastering - 3, Proceedings of the 7th Atlantic Web Intelligence Conference, AWIC 2011, Fribourg, Switzerland, January, 2011, volume 86 of Advances in Intelligent and Soft Computing, pages 163-172. Springer-Verlag, 2011.

9. Jörg Verstraete. Union and intersection of level-2 fuzzy regions. In World Conference on Soft Computing, 2011.

10. Lotfi A. Zadeh. Fuzzy sets. Information and Control, 8:338-353, 1965.

11. Jörg Verstraete, Guy De Tré, Axel Hallez, and Rita De Caluwe. Bitmap based structures for the modelling of fuzzy entities. Control and Cybernetics, 35:147164, 2006.

12. Jörg Verstraete, Guy De Tré, Axel Hallez, and Rita De Caluwe. Using tin-based structures for the modelling of fuzzy gis objects in a database. International Journal of Uncertainty, Fuzziness and Knowledge-Based Systems, 15:1-20, 2007. 